\title{
The Sloan Digital Sky Survey
}

\author{
David W. Hogg \\ Institute for Advanced Study, 1 Einstein Dr., Princeton NJ 08540, USA
}

\begin{abstract}
The Sloan Digital Sky Survey (SDSS) is an imaging and spectroscopic survey of $10^{4} \mathrm{deg}^{2}$ in the North Galactic Cap, currently underway with the 2.5-m SDSS Telescope at the Apache Point Observatory in New Mexico. The data products will include $u, g, r, i$ and $z$-band imaging of several $10^{8}$ sources and $R=2000$ spectra of $10^{6}$ galaxies. The imaging will have 10-sigma limiting (AB) magnitudes of $(u, g, r, i, z)=(21.4,22.6,22.3,21.7,20.1)$. The "Main" sample of $9 \times 10^{5}$ galaxies, selected to have $r<17.7 \mathrm{mag}$, will have spectra with signalto-noise ratio on the order of $\sim 10$ per resolution element, and median redshift $z \sim 0.1$. There will also be a sample of $10^{5}$ luminous elliptical galaxies, selected on the basis of apparent color and magnitude, which will extend out to redshift $z \sim 0.4$. The data will be released steadily over the next five years.

On the order of one percent of the eventual data volume has been assembled at the time of this meeting, with a few hundred square degrees of survey-quality 5-band imaging, and over $10^{4}$ survey-quality spectra. In the refereed literature there are detailed descriptions of the survey design, goals and hardware (York et al 2000; Gunn et al. 1998) and first scientific results (e.g., Ivezic et al 2000; Fischer et al. 2000; Leggett et al. 2000; Yanny et al. 2000).

Relevant to measures of the extragalactic background emission, the survey has found many $z>4$ quasars and has measured the evolution in their space density (e.g., Fan et al. 2000). Measures of bright galaxy counts, the local galaxy luminosity function, and the evolution of elliptical galaxies are in preparation.
\end{abstract}

\section{References}

Fischer, P., et al. 2000, AJ, 120, 1198

Fan, X., et al. 2000, AJ, in press

Gunn, J. E., et al. 1998, AJ, 116, 3040

Ivezic, Z., et al. 2000, AJ, 120, 963

Leggett, S. K., et al. 2000, ApJ, 536, L35

Yanny, B., et al. 2000, ApJ, 540, 825

York, D. G., et al. 2000, AJ, 120, 1579 